\title{
Assessment of unbalance and distortion in power systems that include active power line conditioners.
}

\author{
Jaime Prieto, Patricio Salmerón, Alejandro Pérez, Salvador P. Litrán \\ Department of Electrical Engineering \\ Escuela Técnica Superior de Ingeniería \\ University of Huelva \\ Campus de La Rábida - Palos de la Frontera, 21819 Huelva (Spain) \\ Phone/Fax number:+0034 959217574 / 959217304 \\ E-mail: jpthomas@uhu.es,patricio@uhu.es, aperez@uhu.es, salvador@uhu.es
}

\begin{abstract}
This paper presents a decomposition of the apparent power of a system that allows to separate the contribution to the unbalance of the whole set of harmonics of voltages and currents. From this set of power terms, the unbalanced fundamental, balanced harmonics and unbalanced harmonic indices are derived, that allow to evaluate separately the distortion and the unbalance. This procedure gives a more appropriate way to characterize the mitigation obtained using modern equipments with active compensation. These new indicators of the electric power quality have been applied here to a system with unbalance and distortion in the supply and the load, before and after the connection of a parallel active compensator, and with the connection of a series-parallel active compensator. The results obtained with a dedicated experimental platform have allowed to verify the validity of the new proposed indices.
\end{abstract}

\section{Key words}

Power quality, harmonics, unbalance, measurement, Active Power Filter, Series-Shunt Active Compensation.

\section{Introduction}

The IEEE Std 1459 presents the more extended model nowadays for the decomposition of the apparent power terms, in conditions of asymmetry and distortion, [1]. However, this model does not present an adequate assessment of the unbalance produced by the load, [2]. This is specially significant respect to the contribution to the unbalance introduced by the set of harmonics different from the fundamental. This lack is more accentuated when modern compensation equipment is used. Actually, it is possible to dispose of compensation systems with active power filter, APFs, with parallel connection; and combined systems of series - parallel compensation with the use of APF in series and shunt connection, [3-6]. In this last case, with an appropriated control strategy, it is possible to eliminate the harmonics and unbalance of the current, as well as to obtain a conditioned voltage at the load terminals. The need for the availability of the power terms and the associated indices, to characterize the resultant mitigation, requires a model of decomposition of the apparent power that splits the contribution of the harmonics to the unbalance, [7,8]. In this paper, an alternative decomposition of the apparent power is proposed, in the frame of Std. 1459, that defines four components: the balance fundamental apparent power, the unbalance fundamental apparent power, the balance harmonic apparent power, and the unbalance harmonic apparent power. From these power terms, the unbalance fundamental, balance harmonic, and unbalance harmonic indices are derived; to characterize the unbalance and distortion in a more appropriate way. This new model is applied to a system with unbalance and distortion in the supply and the load, before and after the connection of a parallel active compensator, and with the connection of a series-parallel active compensator. The experimental set up is described and the obtained results are presented, to evaluate the behaviour of the proposed new indices.

\section{Power terms, and power quality indices in Unbalance and Nonsinusoidal Conditions}

In a nonsinusoidal, balanced three-phase system, the harmonics of the phase voltages and currents can be divided into three groups with different sequence of phases. The set of harmonics of order $3 \mathrm{~h}+1$ (with $\mathrm{h}=0$, $1,2, \ldots)$ which have a direct phase sequence, the set of order harmonics $3 \mathrm{~h}+2$, which have an inverse phase sequence, and the set of harmonics $3 \mathrm{~h}+3$, which have an homopolar phase sequence. Therefore, in an unbalanced and nonsinusoidal system, each one of the voltage and current harmonics will have a direct sequence component, a inverse sequence component and a homopolar component. 
Different components of the voltage at the PCC (point of common coupling) of a power system will be defined. A balanced component of the voltage, $\mathrm{Vb}$, is defined, constituted by the phasors of $3 h+1$ order harmonics of direct sequence, $3 \mathrm{~h}+2$ order harmonics of inverse sequence and $3 h+3$ order harmonics of zero sequence. The rms value of this balanced voltage is,

$$
V_{b}^{2}=\sum_{\forall h}\left[\left(V_{d}^{3 h+1}\right)^{2}+\left(V_{i}^{3 h+2}\right)^{2}+\frac{1}{1+\xi}\left(V_{0}^{3 h+3}\right)^{2}\right]
$$

where $\xi$ is taken equal to 1 in the Std. 1459, [6]. The terms $V_{d}, V_{i}$, and $V_{0}$, refer to the components of the direct sequence, inverse and homopolar, respectively, of the corresponding harmonics of the voltage.

The rest of the phasors of the symmetrical components of the harmonics are associated with the component of the unbalanced voltage $V_{u}$, whose rms value is,

$$
\begin{aligned}
& V_{u}^{2}=\sum_{\forall h}\left[\left(V_{i}^{3 h+1}\right)^{2}+\frac{1}{1+\xi}\left(V_{0}^{3 h+1}\right)^{2}+\left(V_{d}^{3 h+2}\right)^{2}+\right. \\
& \left.+\frac{1}{1+\xi}\left(V_{0}^{3 h+2}\right)^{2}+\left(V_{d}^{3 h+3}\right)^{2}+\left(V_{i}^{3 h+3}\right)^{2}\right]
\end{aligned}
$$

In both rms values, $V_{b}$ and $V_{u}$, it is possible to extract the value for the fundamental harmonic. Thus, the fundamental balanced component of the voltage, $V_{b l}$, is

$$
V_{b 1}=V_{b}(h=1) \equiv V_{d}^{1}
$$

And the fundamental unbalanced component, $V_{u l}$,

$$
V_{u 1}=V_{u}(h=1) \equiv \sqrt{\left(V_{i}^{1}\right)^{2}+\frac{1}{1+\xi}\left(V_{0}^{1}\right)^{2}}
$$

From the decomposition of the voltage in four components, the rms value of the voltage (effective voltage), is obtained

$$
V_{e}^{2}=V_{b 1}^{2}+V_{u 1}^{2}+V_{b H}^{2}+V_{u H}^{2}
$$

Similar decomposition is performed with the current. For example, the rms value of the balanced component of the current is,

$$
I_{b}^{2}=\sum_{\forall h}\left[\left(I_{d}^{3 h+1}\right)^{2}+\left(I_{i}^{3 h+2}\right)^{2}+(1+3 \rho)\left(I_{0}^{3 h+3}\right)^{2}\right]
$$

where $\rho$ is the ratio between the neutral conductor resistance and phase conductor resistance; in Std 1459, $\rho=$ 1. Thus, the effective value of the current is,

$$
I_{e}^{2}=I_{b 1}^{2}+I_{u 1}^{2}+I_{b H}^{2}+I_{u H}^{2}
$$

The decomposition established in (5) and (7) to the rms values of the voltage and current, respectively, allows to propose a partition of apparent power that fulfills the relation $(8)$,

$$
\begin{aligned}
& S_{e}^{2}=\left(3 V_{e} I_{e}\right)^{2}= \\
& =S_{b 1}^{2}+S_{u 1}^{2}+S_{b H}^{2}+S_{u H}^{2}
\end{aligned}
$$

which identifies the following four terms of power,

$$
\begin{gathered}
S_{b 1}^{2}=9 V_{b 1}^{2} I_{b 1}^{2} \\
S_{u 1}^{2}=9\left(V_{b 1}^{2} I_{u 1}^{2}+V_{u 1}^{2} I_{b 1}^{2}+V_{u 1}^{2} I_{u 1}^{2}\right) \\
S_{b H}^{2}=9\left(V_{b 1}^{2} I_{b H}^{2}+V_{b H}^{2} I_{b 1}^{2}+V_{b H}^{2} I_{b H}^{2}\right) \\
S_{u H}^{2}=S_{e}^{2}-S_{b 1}^{2}-S_{u 1}^{2}-S_{b H}^{2}
\end{gathered}
$$

$S_{b 1}$ is the balanced fundamental component of apparent power, $S_{u 1}$ is the unbalanced fundamental apparent power, $S_{b H}$ is the balanced harmonic apparent power, and $S_{u H}$ is the unbalanced harmonic apparent power.

If $S_{b l}$ is taken as the base value, it is possible to define indicators related to the unbalance and harmonic distortion in the form,

$$
\begin{gathered}
S U F=\frac{S_{u 1}}{S_{b 1}} \\
S B H=\frac{S_{b H}}{S_{b 1}} \\
S U H=\frac{S_{u H}}{S_{b 1}}
\end{gathered}
$$

$S U F, S B H$ y $S U H$ are Unbalance Fundamental, Balance Harmonic, and Unbalance Harmonic factors of the system, respectively. Similarly, is possible to define global indicators to assess the unbalance and the total harmonic distortion of the system according to the previous,

$$
\begin{aligned}
& S T H D=\sqrt{\frac{S B H^{2}+S U H^{2}}{1+S U F^{2}}} \\
& S T U D=\sqrt{\frac{S U F^{2}+S U H^{2}}{1+S B H^{2}}}
\end{aligned}
$$

STHD and STUD are Total Harmonic Distortion and Total Unbalance Distortion of System, respectively.

In the next section the previous power terms and indexes will be determined for a power system with active compensations in nonsinusoidal and unbalanced conditions.

\section{Experimental Results}

An experimental platform has been set to perform a validation of the proposals and to verify the behaviour of the indices. A parallel and a series - parallel compensation has been applied to an unbalanced and non linear load supplied by an unbalanced set of voltages. Figure 1 shows the power circuit per phase of the series parallel conditioner with their corresponding matching transformers and the passive elements for the filtering of 
the high frequency components. The series and shunt three - phase IGBT converters (Semikron SKM50GB123D) are connected back to back, with a common DC link composed by two electrolytic capacitors, $\mathrm{C}_{\mathrm{DC}+}$ and $\mathrm{C}_{\mathrm{DC}-}$ of $2200 \mu \mathrm{F}, 400 \mathrm{~V}$ each one [4,5]. The middle point of the DC link is connected with the neutral wire of the three phase line. The turn ratio of the matching transformers $T_{P}$ and $T_{S}$ are 1:2 and 2:1 respectively, to achieve reasonable voltage values in the DC link and to set the maximum series compensating voltage at a $25 \%$ of the output voltage of the shunt converter. The filtering inductances $\mathrm{L}_{P}$ and $\mathrm{L}_{\mathrm{S}}$ are of $50 \mathrm{mH}$ each one, and the values of the parallel filtering capacitor $C_{P}$ and resistance $R_{P}$ are $30 \mu F$ and 2.5 $\Omega$ respectively. A panel selector switch allows to bypass the series converter to make tests with only parallel active compensation.

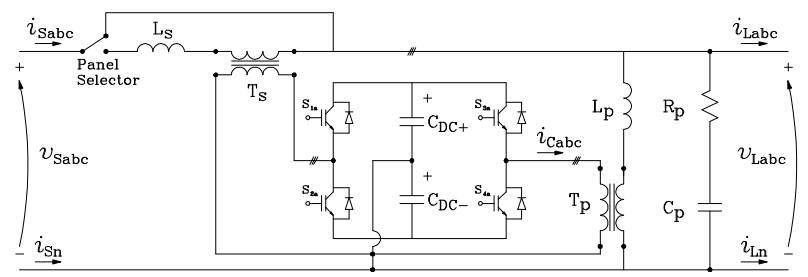

Fig. 1. Power circuit of the active conditioners.

The switching control of the active converters and the calculation of the compensation references are made with a data acquisition and control system (dSPACE-DS1103). The sampling time of the main processor was set to $50 \mu \mathrm{s}$ in the case of the parallel compensation, and to $80 \mu$ s for the series - parallel compensation. The target references for the compensation are the fundamental positive sequence components of the load currents $i_{L}$ and supply voltages $v_{S}$, in order to achieve the lowest unbalance and harmonic components in the compensated system. When only the parallel compensation is performed, the reference for the compensating current $i_{C}$ is:

$$
i_{C}^{*}=i_{L}-i_{S}^{*}
$$

where $i_{S} *$ is a set of balanced currents in phase with the fundamental direct sequence component of the supply voltage, that transports the average active power of the load and the losses of the conditioner [4]. This way, the unbalanced and harmonic components of the load current are compensated.

When the series - parallel compensation is performed, the references for the series compensating voltage $v_{C}$ and the shunt compensating current $i_{C}$ are [4]:

$$
\begin{aligned}
v_{C}^{*} & =\left(v_{L}^{*}-v_{S}\right)+R\left(i_{S}^{*}-i_{S}\right) \\
i_{C}^{*} & =\left(i_{L}-i_{S}^{*}\right)+G\left(v_{L}^{*}-v_{L}\right)
\end{aligned}
$$

where $v_{L} *$ is reference for the load voltage: the fundamental direct sequence component of the supply voltage, regulated in amplitude to its nominal value [4]. The second terms of (19) and (20) are crossed correcting signals proportional to the deviation of the target magnitudes to the reference values, which damp the systems behaviour in strong transients and with different kind of loads. With this kind of compensation, the load voltage $v_{L}$ and the supply current $i_{S}$ should be free of unbalance and harmonic components.

The compensating voltage of the series converter $v_{C}$ is built with a $20 \mathrm{kHz}$ PWM generator (DS1103 slave), using its calculated reference $v_{C}{ }^{*}$. The compensating current of the shunt converters is built with a periodic sampling control at $20 \mathrm{kHz}$, in an external circuit, using the deviation of the compensating current $i_{C}$ respect to its reference $i_{C} *$.

On the other side, an independent measurement system [9] to calculate the indices is implemented in another data acquisition card (dSPACE-DS1005), with a signal conditioning system formed by six voltage sensors (LEM-LV25-P) and eight current sensors (LEM LA35NP). These signals are taken simultaneously to avoid phase differences between the measurements, to enhance the accuracy of the calculated results. The configuration of the virtual instrument was made following the EN 61000-4-7 and EN 61000-4-30 recommendations, using a window equal to five cycles of the fundamental component and a sampling frequency of $6400 \mathrm{~Hz}$ to avoid problems of aliasing and leakage errors.

Finally, the load is composed by three different single phase loads. In the phase 1 it is a single phase diode rectifier with a high capacitor and a resistive load in the dc side, and a smoothing reactor in the ac side. For the phase 2 , it is a single phase diode rectifier with a series RL load in the dc side. And a resistor for the phase 3. This load is fed through a variable autotransformer with an unbalanced set of voltages $(95,100,110 \mathrm{~V})$, considering a nominal value of $100 \mathrm{~V}_{\text {rms }}$, with a small distortion similar to the existing in the supply network of the laboratory. Fig. 2 shows the voltage and current waveforms of the load fed with this power supply.
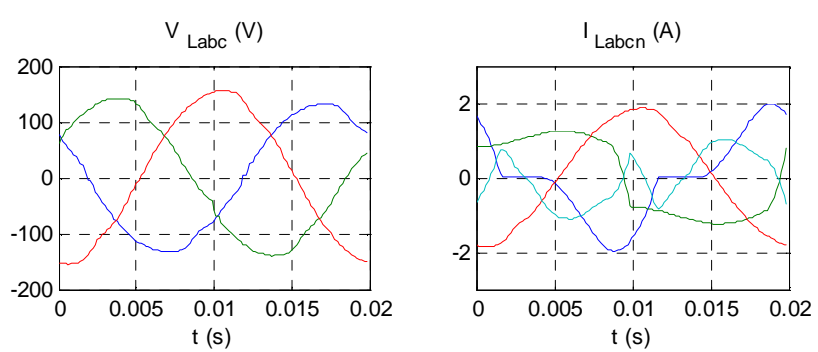

Fig. 2. Voltage and currents waveforms of the load without compensation.

Figures 3 and 4 show the resulting voltage and current waveforms with parallel compensation of the load currents, in the load and supply side respectively. The supply currents $i_{\text {Sabcn }}$ are practically balanced, sinusoidal and in phase with the fundamental positive sequence component of the supply voltage, despite its distortion and unbalance.

Figures 5 and 6 show the resulting voltage and current waveforms with series - parallel compensation of the supply voltages and load currents, in the load and supply 
side respectively. The supply currents are also practically balanced, sinusoidal and in phase with the fundamental positive sequence component of the supply voltage. The load voltage is also enhanced, compensating the distortion and unbalance present in the supply voltage.
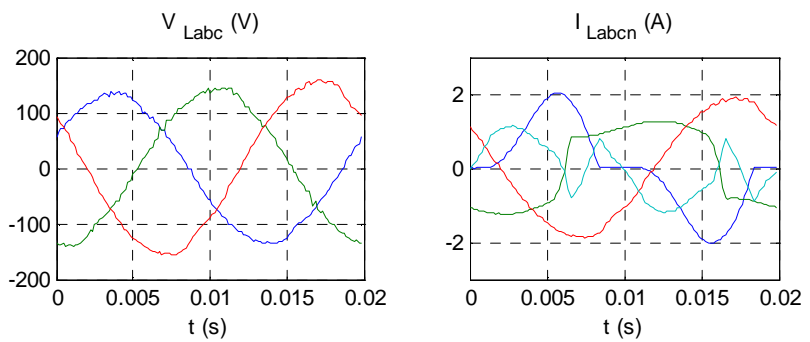

Fig. 3. Voltage and currents waveforms of the load side with active parallel compensation.
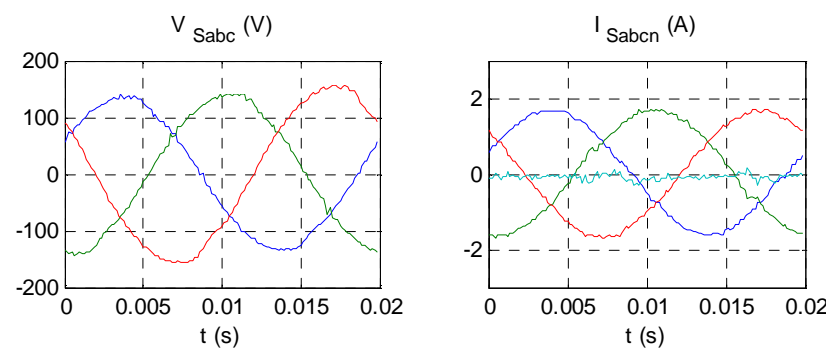

Fig. 4. Voltage and currents waveforms of the supply side with active parallel compensation.
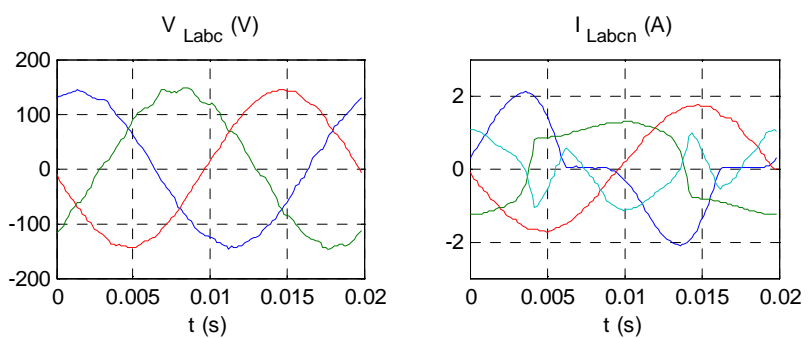

Fig. 5. Voltage and currents waveforms of the load side with active series - parallel compensation.
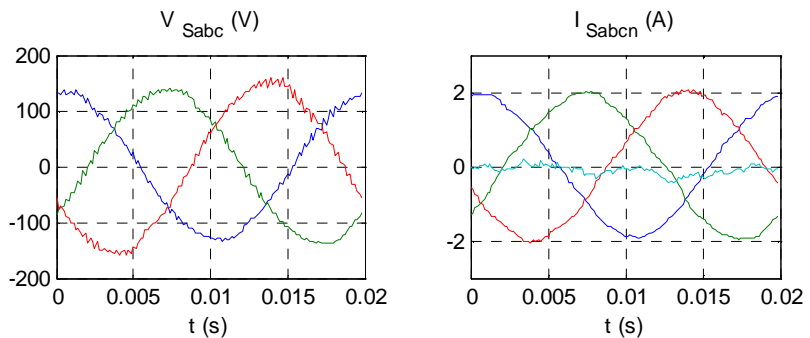

Fig. 6. Voltage and currents waveforms of the supply side with active series - parallel compensation.

Table I presents the calculations obtained with the virtual instrument, corresponding to the effective value of the three-phase voltages, currents and apparent power for the different cases and measuring points: the load without compensation, the voltages and currents of the load side $v_{L}$, $i_{L}$, with parallel compensation (PC_l), the measurements at the supply side $v_{S}, i_{S}$, with parallel compensation (PC_s), and the measurements at the load side and supply side with series - parallel compensation (SPC_1, SPC_s).
Table I. Effective Values of Voltage, Current and Apparent Power

\begin{tabular}{|c|c|c|c|}
\hline & Ve (V) & Ie (A) & Se (VA) \\
\hline Load & 101.2 & 1.206 & 366.1 \\
\hline PC_1 & 101.4 & 1.227 & 373.3 \\
\hline PC_s & 101.4 & 1.166 & 354.9 \\
\hline SPC_1 & 100.7 & 1.201 & 362.9 \\
\hline SPC_s & 99.81 & 1.375 & 411.6 \\
\hline
\end{tabular}

Tables II and III show the values of the different voltage and current components as defined in (5) and (7). It can be seen how the unbalance and harmonic components of the load current are almost compensated with both kinds of compensation; and how the series - parallel compensation produces a regulated load voltage with small unbalance and harmonic components.

Table II. Components of the voltage

\begin{tabular}{|c|c|c|c|c|}
\hline & $\mathrm{V}_{\mathrm{b} 1}(\mathrm{~V})$ & $\mathrm{V}_{\mathrm{u} 1}(\mathrm{~V})$ & $\mathrm{V}_{\mathrm{bH}}(\mathrm{V})$ & $\mathrm{V}_{\mathrm{uH}}(\mathrm{V})$ \\
\hline Load & 101.0 & 5.34 & 2.23 & 2.11 \\
\hline PC_1 & 101.2 & 5.28 & 2.44 & 1.69 \\
\hline PC_s & 101.3 & 5.19 & 2.46 & 1.68 \\
\hline SPC_1 & 100.7 & 0.88 & 1.53 & 2.08 \\
\hline SPC_s & 99.6 & 5.24 & 3.18 & 3.22 \\
\hline
\end{tabular}

Table III. Components of the current

\begin{tabular}{|c|c|c|c|c|}
\hline & $\mathrm{I}_{\mathrm{b} 1}(\mathrm{~A})$ & $\mathrm{I}_{\mathrm{u} 1}(\mathrm{~A})$ & $\mathrm{I}_{\mathrm{bH}}(\mathrm{A})$ & $\mathrm{I}_{\mathrm{uH}}(\mathrm{A})$ \\
\hline Load & 1.075 & 0.399 & 0.257 & 0.271 \\
\hline PC_1 & 1.082 & 0.419 & 0.280 & 0.284 \\
\hline PC_s & 1.164 & 0.020 & 0.034 & 0.052 \\
\hline SPC_1 & 1.066 & 0.380 & 0.282 & 0.287 \\
\hline SPC_s & 1.371 & 0.059 & 0.057 & 0.050 \\
\hline
\end{tabular}

Table IV presents the values of the apparent power components for the different cases and measuring points, with the same structure of the previous tables. In the case of the parallel compensation, it shows a strong reduction of the unbalance fundamental, balanced harmonics and unbalanced harmonics power components in the supply side, due to the enhancement of the supply current. These power components have a small increase at the load side because the parallel compensation can imply a reduction of the filtering effect of the supply impedance [6]. With the series - parallel compensation, the unbalance and harmonic power components at the supply side have also a significant reduction. At the load side the results are similar to the parallel compensation, because the main source of unbalance and distortion are the own load currents. Anyway, a small reduction can be observed in the fundamental unbalance power component due to the enhancement in the load voltage.

Table IV. Apparent Power Components in VA

\begin{tabular}{|c|c|c|c|c|}
\hline & $\mathrm{S}_{\mathrm{b} 1}$ & $\mathrm{~S}_{\mathrm{u} 1}$ & $\mathrm{~S}_{\mathrm{bH}}$ & $\mathrm{S}_{\mathrm{uH}}$ \\
\hline Load & 325.7 & 122.3 & 78.26 & 82.89 \\
\hline PC_1 & 328.5 & 128.6 & 85.50 & 86.81 \\
\hline PC_s & 353.8 & 19.10 & 13.51 & 16.94 \\
\hline SPC_1 & 321.9 & 114.7 & 85.43 & 87.12 \\
\hline SPC_S & 409.6 & 27.91 & 21.42 & 20.15 \\
\hline
\end{tabular}


Table $\mathrm{V}$ presents the corresponding unbalance and harmonic distortion factors (SUF, SBH, SUH) for the different cases. And table VI shows the overall harmonic and unbalance distortions factors (STHD,STUD).

Table V. Unbalance and Harmonic Distortion Factors

\begin{tabular}{|c|c|c|c|}
\hline & SUF (\%) & SBH (\%) & SUH (\%) \\
\hline Load & 37.54 & 24.02 & 25.45 \\
\hline PC_1 & 39.15 & 26.03 & 26.42 \\
\hline PC_s & 5.40 & 3.82 & 4.79 \\
\hline SPC_1 & 35.63 & 26.54 & 27.06 \\
\hline SPC_s & 6.81 & 5.23 & 4.92 \\
\hline
\end{tabular}

Table VI. Overall Harmonic and Unbalance Distortion

\begin{tabular}{|c|c|c|}
\hline & STHD (\%) & STUD (\%) \\
\hline Load & 32.76 & 44.10 \\
\hline PC_1 & 34.53 & 45.71 \\
\hline PC_s & 6.12 & 7.21 \\
\hline SPC_1 & 35.71 & 43.25 \\
\hline SPC_s & 6.81 & 8.39 \\
\hline
\end{tabular}

\section{Conclusions}

A new decomposition of the apparent power in the framework of Std. 1459 has been presented. This partition is based on a rigorous separation of the phase sequence components of the system harmonics. So, as balanced harmonics have been considered those of direct sequence of order $3 h+1$, of inverse sequence of order $3 h+2$ and homopolar sequence of order $3 \mathrm{~h}+3$. These harmonics are the balanced component of the voltage/current. The rest of sequence components of these harmonics form part of the unbalance component of voltage/current.

The above treatment has allowed to propose a partition of apparent power in four terms of power: balance fundamental, unbalance fundamental, balance harmonic and unbalance harmonic of the system. Through the above terms, three indicators of measures of the system EPQ are defined: Unbalance Fundamental, Balance Harmonic and Unbalance Harmonic factors. This way it is possible to evaluate the unbalanced nonsinusoidal situations considering the physical effects of the phenomenon of unbalance in the system. In the same way, these latter indicators were used to introduce two additional indices to assess the distortion and the total unbalance. The new partition of apparent power measures the unbalance introduced by harmonics. This is not possible using the Std-1459. The proposed new model is more appropriated to evaluate the mitigation achieved with active compensation equipments. Effectively, the compensation with parallel APFs allow to actuate over the load current. And by the other side, the compensation with series parallel APFs permits to enhance simultaneously the quality of the voltage and current waveforms. Finally, an experimental platform was developed to test the procedure and the terms of power and indicators introduced. The results showed the viability of the proposed approach.

\section{Acknowledgement}

This work is part of the project "Measurement System for the Identification of Harmonic Distortion Sources and Unbalances in Public Distribution Networks", ref. DPI2010-17709, sponsored by the "Ministerio de Economía y Competitividad" Government of Spain.

\section{References}

[1] "Definitions for the measurement of electric power quantities under sinusoidal, nonsinusoidal, balanced, or unbalanced conditions, IEEE Std. 1459-2000, January 2000.

[2] T. E. Seiphetlho and A. P. J. Rens, "On the assessment of voltage unbalance", 14th International Conference on Harmonics and Quality of Power (ICHQP), 2010, pp. 1-6.

[3] P. Salmerón, J.C. Montaño, J. R. Vázquez, J. Prieto, A. Pérez. "Compensation in nonsinusoidal, unbalanced three-phase four-wire systems with active power-line conditioner". IEEE Transactions on Power Delivery, Vol. 19, No. 4, October 2004, pp. 1968-1974.

[4] J. Prieto, P. Salmerón, J. R. Vázquez, F. J. Alcántara. ""A Series-Parallel Configuration of Active Power Filters for VAR and Harmonic Compensation". Proc. of IECON'02, Sevilla, November 2002, Paper FILTQ.4 pp. 1-6.

[5] J. Prieto, P. Salmerón, R. S. Herrera. "A unified power quality conditioner for wide load range: practical design and experimental results". Proc. of IEEE St. Petersburg PowerTech Conference, 2005, Russia. pp. 429.1 - 429.7.

[6] L. Sainz, J. Balcells. "Harmonic Interaction Influence Due to Current Source Shunt Filters in Networks Supplying Nonlinear Loads", IEEE Trans. on Power Delivery, Vol. 27, No. 3, July 2012, pp. 1385-1393.

[7] T. Zheng, E.B. Makram, A.A. Girgis, "Evaluating power system unbalance in the presence of harmonic distortion". IEEE Trans. on Power Delivery, Vol. 18, No. 2, April 2003, pp. 393397.

[8] G. Chicco, P. Postolache, and C. Toader, "Analysis of threephase systems with neutral under distorted and unbalanced conditions in the symmetrical component-based framework" IEEE Trans. on Power Delivery, Vol. 22, No. 1, January 2007, pp. 674-683.

[9] P. Salmerón, A. Pérez, S. P. Litrán, "New Approach to Assess Unbalance and Harmonic Distortion in Power Systems". Proc. of International Conference on Renewable Energies and Power Quality (ICREPQ'13), Bilbao (Spain), March, 2013, pp. $1-4$. 